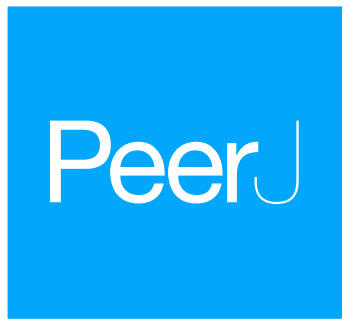

Submitted 18 June 2021

Accepted 20 September 2021

Published 1 November 2021

Corresponding authors

Clément Lozano,

clement.lozano@stir.ac.uk

Sabine Matallana-Surget,

sabine.matallanasurget@stir.ac.uk

Academic editor

Thomas Backhaus

Additional Information and

Declarations can be found on page 7

DOI 10.7717/peerj.12278

(c) Copyright

2021 Lozano et al.

Distributed under

Creative Commons CC-BY 4.0

OPEN ACCESS

\section{Shedding light on the bacterial resistance to toxic UV filters: a comparative genomic study}

\author{
Clément Lozano $^{1,2}$, Philippe Lebaron ${ }^{2}$ and Sabine Matallana-Surget ${ }^{1}$ \\ ${ }^{1}$ Division of Biological and Environmental Sciences, Faculty of Natural Sciences, University of \\ Stirling, Stirling, United Kingdom \\ ${ }^{2}$ Sorbonne Université, CNRS, Laboratoire de Biodiversité et Biotechnologies Microbiennes, \\ USR3579, Observatoire Océanologique, Sorbonne Université, Banyuls-sur-mer, France
}

\section{ABSTRACT}

UV filters are toxic to marine bacteria that dominate the marine biomass. Ecotoxicology often studies the organism response but rarely integrates the toxicity mechanisms at the molecular level. In this study, in silico comparative genomics between UV filters sensitive and resistant bacteria were conducted in order to unravel the genes responsible for a resistance phenotype. The genomes of two environmentally relevant Bacteroidetes and three Firmicutes species were compared through pairwise comparison. Larger genomes were carried by bacteria exhibiting a resistant phenotype, favoring their ability to adapt to environmental stresses. While the antitoxin and CRISPR systems were the only distinctive features in resistant Bacteroidetes, Firmicutes displayed multiple unique genes that could support the difference between sensitive and resistant phenotypes. Several genes involved in ROS response, vitamin biosynthesis, xenobiotic degradation, multidrug resistance, and lipophilic compound permeability were shown to be exclusive to resistant species. Our investigation contributes to a better understanding of UV filters resistance phenotypes, by identifying pivotal genes involved in key pathways.

Subjects Bioinformatics, Genetics, Microbiology, Ecotoxicology, Environmental Impacts Keywords UV filters, Marine bacteria, Genomic comparison

\section{INTRODUCTION}

UV filters are active ingredients of sunscreens products, accounting for up to $30 \%$ in sun lotion formulation. Increasing tourism results in their direct and indirect discharge in aquatic biota, through bathing and wastewater treatment plants (Tovar-Sánchez, SánchezQuiles \& Rodríguez-Romero, 2019). Their bioaccumulation, biomagnification (Lozano et al., 2020a) and their occurrence were reported in freshwater and marine environments from the surface microlayer (Fagervold et al., 2019), water column (Langford et al., 2015; Sánchez Rodríguez, Rodrigo Sanz \& Betancort Rodríguez, 2015) to sediments (Apel, Joerss \& Ebinghaus, 2018; Ramos et al., 2015). UV filters toxicity was demonstrated on a broad range of organisms (Barone et al., 2019; Danovaro et al., 2008; Downs et al., 2016; He et al., 2019; Seoane et al., 2017; Stien et al., 2021; Ziarrusta et al., 2018), including bacteria (Lozano et al., 2020b), yet UV filters toxicity mechanisms remain unclear. Bacteria are an interesting ecotoxicological model as they are easy to handle, constitute a major 
part of the marine biomass (Bar-On, Phillips \& Milo, 2018), and support essential function in marine ecosystems (Yilmaz et al., 2016). In a former study, marine bacteria from the major phyla, namely Actinobacteria, Bacteroidetes, Firmicutes, and Proteobacteria, were sensitive to UV filters from $200 \mu \mathrm{g} / \mathrm{L}$ (Lozano et al., 2020a). Environmentally relevant bacteria investigated in this study belong to the Bacteroidetes, the most abundant phyla after Proteobacteria in marine environments (Coclet et al., 2019), and the Firmicutes, widespread symbionts in marine organisms (Li et al., 2018; Math et al., 2010).

A growing number of bioinformatic tools designed to analyze and compare microbial genomes are available (Karp et al., 2019). They implement genome annotation tools, e.g. RAST, allowing for genome categorization and gene function identification. Functional genomic comparison is widely used to investigate variations between genomes or to depict particular traits (Kube et al., 2013; Wagner et al., 2019). For instance, Kube et al. (2013) provided an extensive functional analysis of the bacterium Oleispira antarctica, corroborating its capacity for alkane degradation and cold adaptation (Kube et al., 2013). Our study aims to perform a genomic comparison of UV filters sensitive and resistant species, using the RAST platform, and the subsystems hierarchical annotation scheme implemented in SEED. Bactericidal compounds can alter numerous cellular functions such as DNA replication, by targeting DNA gyrase (Lewin, Howard \& Smith, 1991), membrane synthesis, by competing with lipid constituting the cell membrane (Müller et al., 2016), or protein synthesis, through binding to ribosomal subunits (Greulich et al., 2015). Although multiple genes might be responsible for bacterial resistance, the targets of UV filters or the membrane permeability towards these compounds are undetermined. Untargeted pairwise comparisons were conducted between resistant and sensitive species, in order to identify genes that could be involved in UV filters resistance and bridge the gap between genotypes and phenotypic traits. Comparisons were performed to address relevant functions, such as membrane biosynthesis, cell signaling, stress response and xenobiotic degradation.

\section{MATERIALS AND METHODS}

\section{Bacterial strain genomes}

Genomes of bacterial species screened for sensitivity (Lozano et al., 2020a) were downloaded from NCBI (https://www.ncbi.nlm.nih.gov/genome). The following strains from comparable phyla and genera were selected based on their resistance profiles: Paenibacillus glucanolyticus NBRC 15330, Bacillus megaterium ATCC 14581, Halobacillus dabanensis CGMCC 1.3704, Algoriphagus ornithinivorans DSM 15282, and Algoriphagus mannitolivorans DSM 15301.

\section{Genome comparison}

Bacterial genomes were uploaded on the RAST platform (https://rast.nmpdr.org). Subsequently, pairwise comparisons were conducted between sensitive and resistant bacteria belonging to the same Phylum (Table 1), using the SEED functional classification. Average nucleotide identity (ANI) between the compared species were calculated using the 
Table 1 Pairwise compared species and respective UV filters sensitivity.

\begin{tabular}{lllll} 
Phyla & Species & Sensitive/resistant (UV filters) & ANI Score (\%) \\
\hline Bacteroidetes & Algoriphagus mannitolivorans & $\mathrm{R}$ & (HS) & 72.13 \\
Bacteroidetes & Algoriphagus ornithinivorans & $\mathrm{S}$ & & \\
Firmicutes & Paenibacillus glucanolyticus & $\mathrm{S}$ & (EHMC) & 65.32 \\
Firmicutes & Halobacillus dabanensis & $\mathrm{S}$ & (EHMC, HS) & \\
Firmicutes & Bacillus megaterium & $\mathrm{R}$ & (EHMC, HS) & 68.02 \\
Firmicutes & Halobacillus dabanensis & $\mathrm{S}$ & & \\
Note: & &
\end{tabular}

\begin{tabular}{|c|c|c|c|c|c|c|c|c|}
\hline Species & Chromosome & Plasmid & Size (Mb) & GC\% & Genes & rRNA & tRNA & Proteins \\
\hline Algoriphagus mannitolivorans* & 1 & 0 & 4.15 & 42.7 & 3636 & 7 & 39 & 3564 \\
\hline Algoriphagus ornithinivorans & 1 & 0 & 4.09 & 39.5 & 3,557 & 5 & 35 & 3,500 \\
\hline Paenibacillus glucanolyticus* & 1 & 0 & 5.9 & 49.2 & 5,380 & 24 & 74 & 5,174 \\
\hline Halobacillus dabanensis & 1 & 0 & 4.14 & 41.7 & 4,135 & 18 & 67 & 3,971 \\
\hline Bacillus megaterium* & 1 & 6 & 5.34 & 38.1 & 5,541 & 41 & 123 & 5,268 \\
\hline
\end{tabular}

Note:

Asterisks $\left({ }^{*}\right)$ indicate UV filters resistant bacteria.

EZbiocloud platform (https://www.ezbiocloud.net) to provide a view of the species relativeness.

\section{Protein comparison}

Protein FASTA files were downloaded from the RAST platform and uploaded on the Orthovenn online tool (https://orthovenn2.bioinfotoolkits.net/) to generate Venn diagrams.

\section{RESULTS AND DISCUSSION}

\section{Bacterial genome size as an indicator of bacterial resistance}

Bacteroidetes selected in this study-belonging to the Algoriphagus genera-exhibited a smaller genome compared to Firmicutes (Table 2). Similarly, the number of rRNA, tRNA, and proteins was lower in the Bacteroidetes species. Among Firmicutes, H. dabanensis displayed the smaller genome with approximately 1,000 fewer proteins than B. megaterium and P. glucanolyticus. Noteworthy, UV filters resistant species for both Firmicutes and Bacteroidetes held the greater genome. A correlation between the appearance of resistance mechanisms and genome size has already been discussed earlier (Projan \& Levy, 2007). Similarly, a high tRNA number has been correlated with temperature resistance (Siddhartha Sankar, Malay \& Suvendra Kumar, 2010).

A. mannitolivorans and A. ornithinivorans shared 2,823 proteins and displayed 34 and 28 unique proteins, respectively (Fig. 1A). Among these proteins, A. mannitolivorans displayed two protein counts included in the "cellular aromatic compound metabolic process" cluster, identified as the 4,5-DOPA dioxygenase extradiol, an enzyme involved in 
A

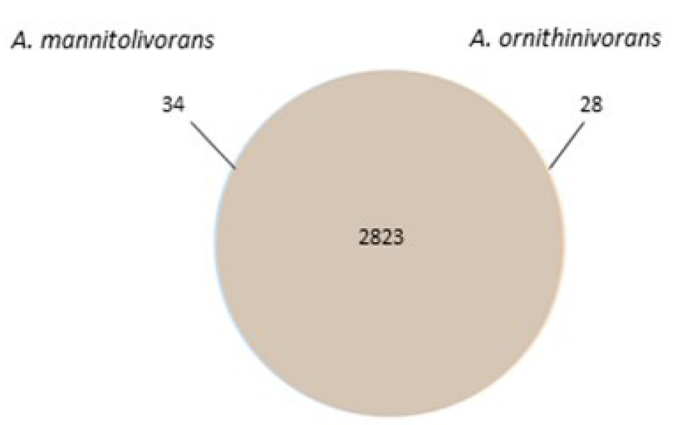

C B. megaterium
B

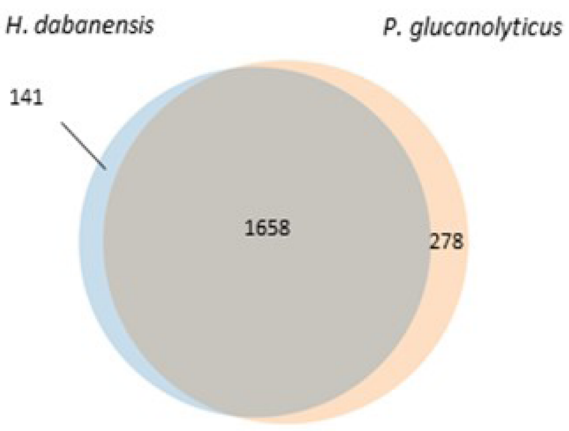

H. dabanensis

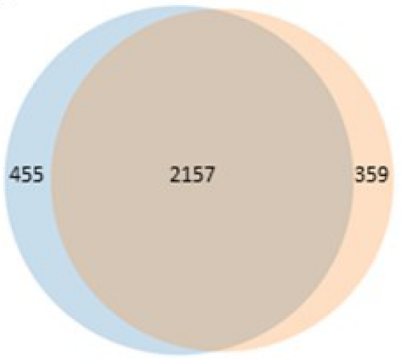

Figure 1 Venn diagrams representing the number of unique and shared proteins between (A) A. mannitolivorans and A. ornithinivorans, (B) $H$. dabanensis and $P$. glucanolyticus, (C) B. megaterium and H. dabanensis. Full-size $\underset{\bullet}{\bullet}$ DOI: $10.7717 /$ peerj.12278/fig-1

betalain biosynthesis. P. glucanolyticus and $H$. dabanensis shared 1,658 proteins (Fig. 1B). B. megaterium and $H$. dabanensis shared 2,157 proteins and exhibited 455 and 359 unique proteins, respectively (Fig. 1C). Interestingly bacteria displaying the greater number of unique proteins held the resistant phenotype.

\section{Comparative genomic analysis of $\boldsymbol{H}$. dabanensis and $B$. megaterium revealed relevant resistance features}

The cell wall constitutes the first barrier against xenobiotics. Therefore, genes classified in this functional category were compared in this study. B. megaterium displayed 14 unique genes belonging to the cell wall category while $H$. dabanensis had only one. Among them, the subsystems "Rhamnose containing glycans" and "sialic acid metabolism" were the most represented. Rhamnose is a six-carbon deoxy hexose, mostly incorporated in cell wall anchored polysaccharides, glycoproteins, and the capsule of many bacteria (Mistou,

Sutcliffe \& Van Sorge, 2016). It is essential for cell viability, environmental adaptation, and biofilm formation (Mäki \& Renkonen, 2004; Michael et al., 2016). The presence of genes involved in rhamnose metabolism and the gene coding for the capsular polysaccharide biosynthesis protein strongly suggests that $B$. megaterium holds a capsule on its surface (see Fig. 2 for a schematic representation). While phospholipid bilayers are hypothetically permeable to lipophilic compounds such as homosalate, the capsule, made of a polysaccharides layer could prevent these compounds from entering the cell. Sialic acids are nine carbon sugar acids derivatives mainly incorporated into glycan chains. Common 


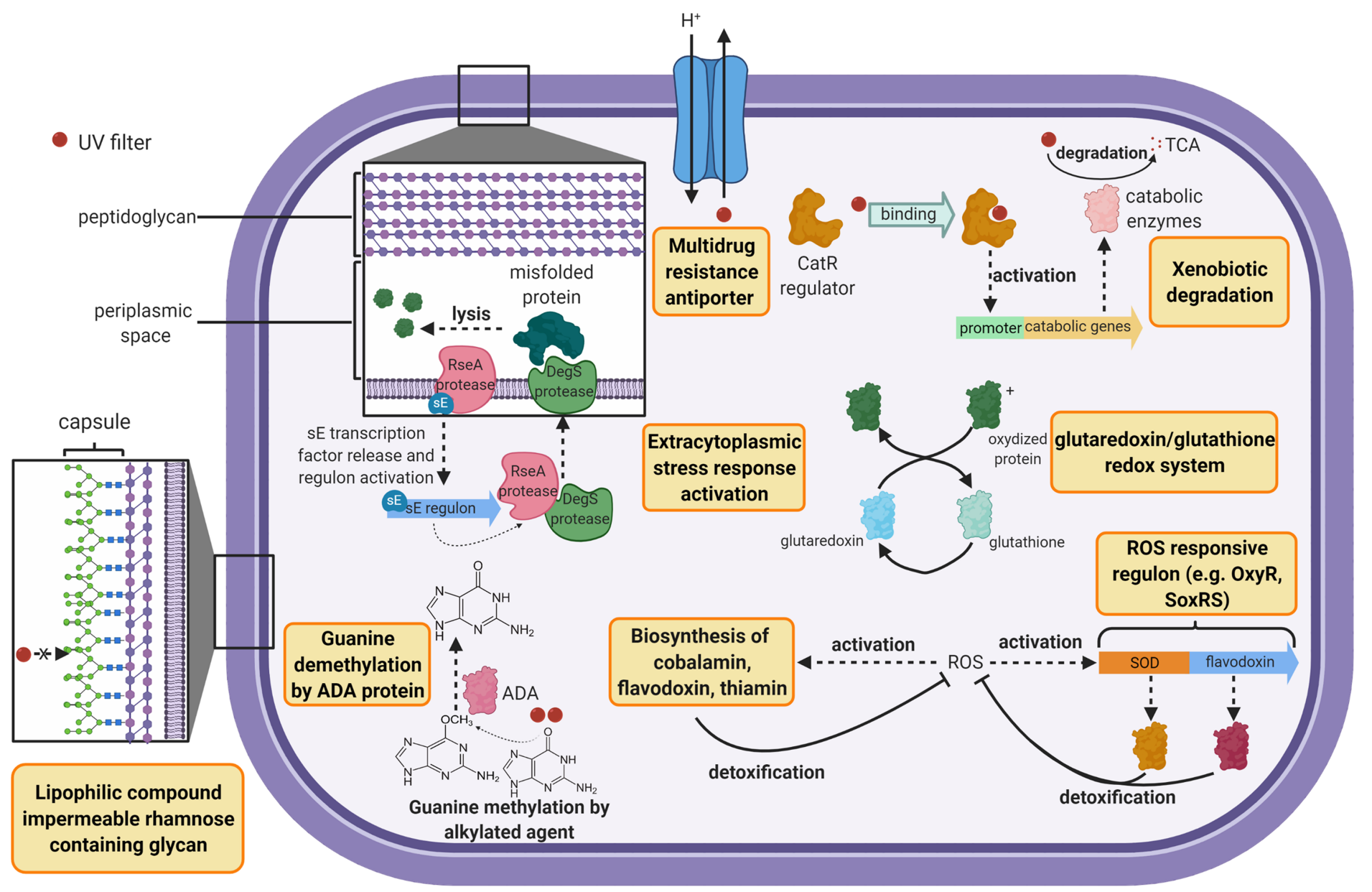

Figure 2 Cell diagram showing the hypothetical protective structures against UV filters, obtained from genomic functional comparison.

Full-size $\square$ DOI: 10.7717 /peerj.12278/fig-2

on the surface of pathogenic bacteria, they provide camouflage from the immune system by imitating eukaryotic structures.

Both species harbored genes involved in bacitracin response. B. megaterium possessed multiple stress response genes such as cadmium-transporting ATPase, multicopper oxidase and multidrug resistance transporter (Bcr/CflA family), the cytoplasmic copper homeostasis protein $\mathrm{CutC}$, the mercuric resistance operon regulatory protein, and the membrane fusion protein of RND family multidrug efflux pump, known to confer resistance against multiple hydrophobic substrates (Nikaido \& Takatsuka, 2009). Several gene copies belonging to the choline and betaine uptake and biosynthesis subsystem were observed in the genome of B. megaterium, including four choline specific $A B C$ transporter. Choline is a precursor of glycine betaine, known as a potent osmoprotectant (Kiene, 1998). Furthermore, B. megaterium presented genes coding for flavodoxin, a small soluble protein belonging to the non-enzymatic antioxidant molecules, known to confer resistance against herbicides to soil bacteria (Coba de la Pena et al., 2013) (Fig. 2). Among the genes categorized in the regulation and cell signaling category, the aromatic 
hydrocarbon utilization transcriptional regulator CatR (LysR family) was the single unique gene belonging to $B$. megaterium. CatR regulates the expression of catechol and phenol degradation pathway, that has an aromatic structure (benzene) common to UV filters (Díaz \& Prieto, 2000). Once the substrate binds to CatR, the promoter regulating the transcription of the catabolic genes is activated and the substrate is degraded into tricarboxylic acid intermediates (Díaz \& Prieto, 2000) (Fig. 2).

\section{Comparative genome analysis of $\boldsymbol{H}$. dabanensis and Paenibacillus glucanolyticus}

Compared to the UV filter sensitive H. dabanensis, P. glucanolyticus harbored more genes coding for proteins involved in pyridoxine (vitamin B6), cobalamin (vitamin B12), and thiamin (vitamin B1) biosynthesis. Vitamins, such as vitamin B12 allow tolerance to oxidative stress in bacteria belonging to the Nitrospira phylum (Ferrer et al., 2016). Similarly to B. megaterium, P. glucanolyticus coded for Hyaluronan synthase and all genes belonging to the Rhamnose containing glycans subsystem, involved in capsule synthesis - a structure known to mediate antibiotic resistance (Campos et al., 2004). A total of 11 unique genes categorized in subsystem related to antibiotic and metal stress response were identified in the genomes of $P$. glucanolyticus. Among them, the Multidrug resistance transporter (Bcr/CflA family), reported being involved in bicyclomycin, fosfomycin, kanamycin, and sulfathiazole resistance (Smith, Kumar \& Varela, 2009). Transcriptomic analyses highlighted that this transporter was overexpressed ( 4.35 fold change) in the presence of toluene (García et al., 2010) in Pseudomonas putida, hence revealing that this gene could play a pivotal role in xenobiotic resistance.

Both bacteria harbored DNA repair systems such as Rec, SOS response, and mutL-mutS system. $P$. glucanolytics expressed the ADA regulatory protein, involved in six-OMethylguanine demethylation, a mechanism known to bypass $\mathrm{G}: \mathrm{C}$ to A:T due to guanine methylation (Fig. 2). P. glucanolyticus genome contained multiple genes involved in response against oxidative stress such as superoxide dismutase, HtrA protease/chaperone protein, Glutaredoxin, and the outer membrane stress sensor protease DegS. HtrA protease/chaperone protein was shown to be involved in high temperature and oxidative stress tolerance in Campylobacter jejuni (Beek et al., 2011). Mutation induced lack of glutathione-glutaredoxin in a Rhodobacter strain resulted in decreased growth rates and high sensitivity to oxidative stress (Li et al., 2004). In addition, proteomic analyses revealed that glutaredoxin was up-regulated in Rhodobacter sp. exposed to artificial UVB treatment (Pérez et al., 2017). Overall, we can postulate that among Firmicutes, both P. glucanolyticus, and B. megaterium were better fitted to cope with the potentially harmful effect of xenobiotics, including UV filters, than $H$. dabanensis.

\section{Algoriphagus displayed fewer genomics differences but different phenotypes}

The UV filter resistant A. mannitolivorans showed a chromosomic Doc/Phd toxin-antitoxin system, known to help cells maintaining genome integrity over generations, control cell growth, and face environmental stresses, by entering into 
persistent states (Liu et al., 2008). Interestingly, A. mannitolivorans genome harbored the gene coding for the antitoxin ParD without the corresponding DNA gyrase targeting toxin, ParE. Therefore, we could hypothesize that ParD can compete WITH for other DNA gyrase binding molecules. DNA repair systems were similar between the two species. However, A. ornithinivorans showed five genes belonging to the CRISPR system, involved in bacterial prophage immunity.

A. ornithinivorans and A. mannitolivorans showed genes involved in the resistance against Beta-lactam, Bacitracin, Fosfomycin, fluoroquinolones, and metal such as cadmium, cobalt, and copper. Genes coding for multiple multidrug resistance efflux pumps were present in both species. Minor differences were observed between genes classified into the cell wall and capsule category. No genes involved in xenobiotics resistance were identified in the two strains. As discussed earlier, these two species displayed only a few unique proteins (Fig. 1A). Overall, these observations suggested that only minor genomic differences could explain different phenotypes.

\section{CONCLUSION}

Phenotypes variations were supported by genomic pairwise comparisons between Firmicutes and Bacteroidetes species. Overall, the analysis of Bacteroidetes genomes displayed two distinctive features, i.e. the antitoxin and CRISPR systems. On the other hand, the analysis of Firmicutes genomes allowed for the identification of multiple genes that could be involved in UV filters resistant mechanisms, coding for multidrug transporters, ROS responsive elements, periplasmic stress response regulons, or capsule components. Interestingly, bacteria with the bigger genomes held the resistant phenotypes, corroborating the fact that organisms with larger genomes are more adaptable to environmental and anthropogenic perturbations. While the presence of genes has been studied to decipher potential resistance mechanisms, the absence of a gene or operon could also be responsible for resistance by depleting the molecular target of UV filters. Further functional experiments such as transcriptomic and proteomic analyses would be needed to confirm the xenobiotic susceptibility and resistance biomarkers.

\section{ADDITIONAL INFORMATION AND DECLARATIONS}

\section{Funding}

This work was supported by the Carnegie Research Incentive Grant (RIG0088612). Clement Lozano is the recipient of a 50/50 match funding scholarship between the University of Stirling (Scotland, UK) and Sorbonne University (Paris, France). The funders had no role in study design, data collection and analysis, decision to publish, or preparation of the manuscript.

\section{Grant Disclosures}

The following grant information was disclosed by the authors:

Carnegie Research Incentive Grant: RIG0088612. 
University of Stirling.

Sorbonne University.

\section{Competing Interests}

The authors declare that they have no competing interests.

\section{Author Contributions}

- Clément Lozano conceived and designed the experiments, performed the experiments, analyzed the data, prepared figures and/or tables, authored or reviewed drafts of the paper, and approved the final draft.

- Philippe Lebaron conceived and designed the experiments, authored or reviewed drafts of the paper, and approved the final draft.

- Sabine Matallana-Surget conceived and designed the experiments, analyzed the data, authored or reviewed drafts of the paper, and approved the final draft.

\section{Data Availability}

The following information was supplied regarding data availability:

The genomic pairwise comparison obtained from the RAST platform (rast.nmpdr.org) are available in the Supplemental Files.

\section{Supplemental Information}

Supplemental information for this article can be found online at http://dx.doi.org/10.7717/ peerj.12278\#supplemental-information.

\section{REFERENCES}

Apel C, Joerss H, Ebinghaus R. 2018. Environmental occurrence and hazard of organic UV stabilizers and UV filters in the sediment of European North and Baltic Seas. Chemosphere 212:254-261 DOI 10.1016/j.chemosphere.2018.08.105.

Bæk KT, Vegge CS, Skórko-Glonek J, Brøndsted L. 2011. Different contributions of HtrA protease and chaperone activities to Campylobacter jejuni stress tolerance and physiology. Applied and Environmental Microbiology 77(1):57-66 DOI 10.1128/AEM.01603-10.

Bar-On YM, Phillips R, Milo R. 2018. The biomass distribution on earth. Proceedings of the National Academy of Sciences of the United States of America 115(25):6506-6511 DOI 10.1073/pnas.1711842115.

Barone AN, Hayes CE, Kerr JJ, Lee RC, Flaherty DB. 2019. Acute toxicity testing of $\mathrm{TiO}_{2}$-based vs. oxybenzone-based sunscreens on clownfish (Amphiprion ocellaris). Environmental Science and Pollution Research 26(14):14513-14520 DOI 10.1007/s11356-019-04769-z.

Campos MA, Vargas MA, Baleares I, De Mallorca P. 2004. Capsule polysaccharide mediates bacterial resistance to antimicrobial peptides. Infection and Immunity 72(12):7107-7114 DOI 10.1128/IAI.72.12.7107.

Coba de la Pena T, Redondo FJ, Fillat M, Lucas M, Pueyo JJ. 2013. Flavodoxin overexpression confers tolerance to oxidative stress in beneficial soil bacteria and improves survival in the presence of the herbicides paraquat and atrazine. Journal of Applied Microbiology 115(1):236-246 DOI 10.1111/jam.12224.

Coclet C, Garnier C, Durrieu G, Omanović D, D’Onofrio S, Le Poupon C, Mullot J-U, Briand JF, Misson B. 2019. Changes in bacterioplankton communities resulting from direct and indirect 
interactions with trace metal gradients in an urbanized marine coastal area. Frontiers in Microbiology 10:77 DOI 10.3389/fmicb.2019.00257.

Danovaro R, Bongiorni L, Corinaldesi C, Giovannelli D, Damiani E, Astolfi P, Greci L, Pusceddu A. 2008. Sunscreens cause coral bleaching by promoting viral infections. Environmental Health Perspectives 116(4):441-447 DOI 10.1289/ehp.10966.

Díaz E, Prieto MA. 2000. Bacterial promoters triggering biodegradation of aromatic pollutants. Current Opinion in Biotechnology 11(5):467-475 DOI 10.1016/S0958-1669(00)00126-9.

Downs CA, Kramarsky-Winter E, Segal R, Fauth J, Knutson S, Bronstein O, Ciner FR, Jeger R, Lichtenfeld Y, Woodley CM, Pennington P, Cadenas K, Kushmaro A, Loya Y. 2016. Toxicopathological effects of the sunscreen UV filter, oxybenzone (Benzophenone-3), on coral planulae and cultured primary cells and its environmental contamination in Hawaii and the U.S. Virgin Islands. Archives of Environmental Contamination and Toxicology 70(2):265-288 DOI 10.1007/s00244-015-0227-7.

Fagervold SK, Rodrigues AS, Rohée C, Roe R, Bourrain M, Stien D, Lebaron P. 2019. Occurrence and environmental distribution of 5 UV filters during the summer season in different water bodies. Water Air Soil Pollution 3(230):172 DOI 10.1007/s11270-019-4217-7.

Ferrer A, Rivera J, Zapata C, Norambuena J, Sandoval Á, Chávez R, Levicán G, Orellana O. 2016. Cobalamin protection against oxidative stress in the acidophilic iron-oxidizing bacterium Leptospirillum Group II CF-1. Frontiers in Microbiology 7:1-11 DOI 10.3389/fmicb.2016.00748.

García V, Godoy P, Daniels C, Hurtado A, Ramos JL, Segura A. 2010. Functional analysis of new transporters involved in stress tolerance in Pseudomonas putida DOT-T1E. Environmental Microbiology Reports 2(3):389-395 DOI 10.1111/j.1758-2229.2009.00093.x.

Greulich P, Scott M, Evans MR, Allen RJ. 2015. Growth-dependent bacterial susceptibility to ribosome-targeting antibiotics. Molecular Systems Biology 11(3):796

DOI 10.15252/msb.20145949.

He T, Tsui MMP, Tan CJ, Ng KY, Guo FW, Wang LH, Chen TH, Fan TY, Lam PKS, Murphy MB. 2019. Comparative toxicities of four benzophenone ultraviolet filters to two life stages of two coral species. Science of the Total Environment 651:2391-2399

DOI 10.1016/j.scitotenv.2018.10.148.

Karp PD, Ivanova N, Krummenacker M, Kyrpides N. 2019. A comparison of microbial genome web portals. Frontiers in Microbiology 10(February):1-13 DOI 10.3389/fmicb.2019.00208.

Kiene RP. 1998. Uptake of choline and its conversion to glycine betaine by bacteria in estuarine waters. Applied and Environmental Microbiology 64(3):1045-1051.

Kube M, Chernikova TN, Al-Ramahi Y, Beloqui A, Lopez-Cortez N, Guazzaroni Mía-E, Heipieper HJ, Klages S, Kotsyurbenko OR, Langer I, Nechitaylo TY, Lünsdorf H, Fernández M, Juárez S, Ciordia S, Singer A, Kagan O, Egorova O, Alain Petit P, Stogios $P$, Kim Y, Tchigvintsev A, Flick R, Denaro R, Genovese M, Albar JP, Reva ON, MartínezGomariz M, Tran H, Ferrer M, Savchenko A, Yakunin AF, Yakimov MM, Golyshina OV, Reinhardt R, Golyshin PN. 2013. Genome sequence and functional genomic analysis of the oil-degrading bacterium Oleispira antarctica. Nature Communications 4(1):201 DOI 10.1038/ncomms3156.

Langford KH, Reid MJ, Fjeld E, Øxnevad S, Thomas KV. 2015. Environmental occurrence and risk of organic UV filters and stabilizers in multiple matrices in Norway. Environment International 80:1-7 DOI 10.1016/j.envint.2015.03.012.

Lewin CS, Howard BMA, Smith JT. 1991. Protein-and RNA-synthesis independent bactericidal activity of ciprofloxacin that involves the A subunit of DNA gyrase. Journal of Medical Microbiology 34:19-22. 
Li K, Hein S, Zou W, Klug G. 2004. The glutathione-glutaredoxin system in Rhodobacter capsulatus: part of a complex regulatory network controlling defense against oxidative stress. Journal of Bacteriology 186(20):6800-6808 DOI 10.1128/JB.186.20.6800-6808.2004.

Li Y-F, Yang N, Liang X, Yoshida A, Osatomi K, Power D, Batista FM, Yang J-L. 2018. Elevated seawater temperatures decrease microbial diversity in the gut of Mytilus coruscus. Frontiers in Physiology 9(July):1-9 DOI 10.3389/fphys.2018.00839.

Liu M, Zhang Y, Inouye M, Woychik NA. 2008. Bacterial addiction module toxin Doc inhibits translation elongation through its association with the $30 \mathrm{~S}$ ribosomal subunit. Proceedings of the National Academy of Sciences of the United States of America 105(15):5885-5890 DOI 10.1073/pnas.0711949105.

Lozano C, Givens J, Stien D, Matallana Surget S, Lebaron P. 2020a. Bioaccumulation and toxicological effects of UV-filters on marine species. In: Handbook of Environmental Chemistry. Berlin: Springer.

Lozano C, Matallana-surget S, Givens J, Nouet S, Arbuckle L, Lambert Z, Lebaron P. 2020 b. Toxicity of UV filters on marine bacteria : combined effects with damaging solar radiation. Science of the Total Environment 722(10):137803 DOI 10.1016/j.scitotenv.2020.137803.

Mäki M, Renkonen R. 2004. Biosynthesis of 6-deoxyhexose glycans in bacteria. Glycobiology 14(3):1R-15 DOI 10.1093/glycob/cwh040.

Math RK, Islam SMA, Hong SJ, Cho KM, Kim JM, Yun MG, Cho JJ, Kim EJ, Lee YH, Yun HD. 2010. Metagenomic characterization of oyster shell dump reveals predominance of firmicutes bacteria. Microbiolgy 79(4):509-519 DOI 10.1134/S0026261710040132.

Michael V, Frank O, Bartling P, Scheuner C, Göker M. 2016. Biofilm plasmids with a rhamnose operon are widely distributed determinants of the 'swim-or-stick' lifestyle in roseobacters. ISME Journal 10(10):2498-2513 DOI 10.1038/ismej.2016.30.

Mistou M, Sutcliffe IC, Van Sorge NM. 2016. Bacterial glycobiology : rhamnose-containing cell wall polysaccharides in gram-positive bacteria. FEMS Microbiology Reviews 40()):464-479 DOI 10.1093/femsre/fuw006.

Müller A, Wenzel M, Strahl H, Grein F, Saaki TNV, Kohl B, Siersma T, Bandow JE, Sahl H-G, Schneider T, Hamoen LW. 2016. Daptomycin inhibits cell envelope synthesis by interfering with fluid membrane microdomains. Proceedings of the National Academy of Sciences of the United States of America 113(45):E7077-E7086 DOI 10.1073/pnas.1611173113.

Nikaido H, Takatsuka Y. 2009. Mechanisms of RND multidrug efflux pumps. Physical Review C 51(6):769-781 DOI 10.1103/PhysRevC.51.3256.

Pérez V, Hengst M, Kurte L, Dorador C, Jeffrey WH, Wattiez R, Molina V, Matallana-Surget S. 2017. Bacterial survival under extreme UV radiation : a comparative proteomics study of rhodobacter sp., isolated from high altitude wetlands in chile bacterial isolation and growth. Frontiers in Microbiology 8(June):1-16 DOI 10.3389/fmicb.2017.01173.

Projan SJ, Levy S. 2007. (Genome) size matters. Antimicrobial Agents and Chemotherapy 51(4):1133-1134 DOI 10.1128/AAC.01370-06.

Ramos S, Homem V, Alves A, Santos L. 2015. Advances in analytical methods and occurrence of organic UV-filters in the environment-a review. Science of the Total Environment 526:278-311 DOI 10.1016/j.scitotenv.2015.04.055.

Sánchez Rodríguez A, Rodrigo Sanz M, Betancort Rodríguez JR. 2015. Occurrence of eight UV filters in beaches of Gran Canaria (Canary Islands). An approach to environmental risk assessment. Chemosphere 131(Suppl):85-90 DOI 10.1016/j.chemosphere.2015.02.054. 
Seoane M, Esperanza M, Rioboo C, Herrero C, Cid Á. 2017. Flow cytometric assay to assess short-term effects of personal care products on the marine microalga Tetraselmis suecica. Chemosphere 171(15):339-347 DOI 10.1016/j.chemosphere.2016.12.097.

Siddhartha Sankar S, Malay D, Suvendra Kumar R. 2010. Higher tRNA diversity in thermophilic bacteria: a possible adaptation to growth at high temperature. Microbiological Research 165(8):609-616.

Smith KP, Kumar S, Varela MF. 2009. Identification, cloning, and functional characterization of EmrD-3, a putative multidrug efflux pump of the major facilitator superfamily from Vibrio cholerae O395. Archives of Microbiology 191(12):903-911 DOI 10.1007/s00203-009-0521-8.

Stien D, Suzuki M, Rodrigues AMS, Yvin M, Clergeaud F, Thorel E, Lebaron P. 2021. A unique approach to monitor stress in coral exposed to emerging pollutants. Scientific Reports 10(1):1-12 DOI 10.1038/s41598-020-66117-3.

Tovar-Sánchez A, Sánchez-Quiles D, Rodríguez-Romero A. 2019. Massive coastal tourism influx to the Mediterranean Sea: the environmental risk of sunscreens. Science of the Total Environment 656:316-321 DOI 10.1016/j.scitotenv.2018.11.399.

Wagner J, Harrison EM, Martinez Del Pero M, Blane B, Mayer G, Leierer J, Gopaluni S, Holmes MA, Parkhill J, Peacock SJ, Jayne DRW, Kronbichler A. 2019. The composition and functional protein subsystems of the human nasal microbiome in granulomatosis with polyangiitis: a pilot study. Microbiome 7(137):1-17 DOI 10.1186/s40168-019-0753-z.

Yilmaz P, Yarza P, Rapp JZ, Glöckner FO. 2016. Expanding the world of marine bacterial and archaeal clades. Frontiers in Microbiology 6(JAN):1-29 DOI 10.3389/fmicb.2015.01524.

Ziarrusta H, Mijangos L, Picart-Armada S, Irazola M, Perera-Lluna A, Usobiaga A, Prieto A, Etxebarria N, Olivares M, Zuloaga O. 2018. Non-targeted metabolomics reveals alterations in liver and plasma of gilt-head bream exposed to oxybenzone. Chemosphere 211:624-631 DOI 10.1016/j.chemosphere.2018.08.013. 